\title{
Research Progress of Quartz Tuning Fork Micromachined Gyroscope
}

\author{
Maoyan Fan \\ School of Information Technology and Engineering \\ Yuxi Normal University \\ Yuxi, China \\ e-mail: fmy@yxnu.net
}

\author{
Lifang Zhang \\ Office of Academic Affairs \\ Yuxi Normal University \\ Yuxi, China \\ e-mail: ff@yxnu.net
}

\begin{abstract}
This paper presents a review of the working principle, the development and the research progress of the quartz tuning fork micromachined gyroscopes. Then, the fabrication process, the performance parameters and the application fields of the QRS are illustrated. Next, the development trends and the existing problems are analyzed, including the uneven crystal edges of the quartz sidewall caused by the fabrication process, the confined etchant layer technology and the electrode fabrication technique. In summary, the QRSs are widely applied in many fields, including military and commercial applications, because of its small size, lightweight, low power consumption, high resistance to overload and availability in harsh environments.
\end{abstract}

Keywords-Coriolis acceleration; piezoelectric effect; quartz tuning fork; micromachined gyroscope; anisotropic etching

\section{INTRODUCTION}

The quartz tuning fork micromachined gyroscope (QRS) is a kind of inertial sensors which are sensitive to the angular rate and plays a very important role in the flight attitude control, guidance and positioning and so on. Recently it is a prevailing trend for the guidance of conventional weapons and the miniaturization of tactical guided weapons. The low-cost micro inertial guidance system can achieve the guidance of unguided munitions, increase the hitting accuracy, and effectively enhance the destruction effect and the lethality of weapons. The QRS has many advantages such as small size, lightweight, low power consumption, exceptional stability over temperature, high resistance to overload and availability in harsh environments and the medium and low precision.

\section{WORKING PRINCIPLE}

The quartz tuning fork micromachined gyroscopes are an example of vibratory gyroscopes. Its operation principle is based on the Coriolis effect in the rotation dynamics and utilizing the piezoelectric effect of quartz to sense Coriolis force [1,2]. The quartz tuning fork gyroscopes are divided into the single tuning fork and the double tuning forks. The single tuning fork drives and senses with only one fork, so it is very simple in both structural design and processing. The double tuning forks comprise the drive mode and the sense mode with different functions and which are able to achieve decoupling of the drive and the sense signals. Figure 1 shows the structure model of the micromachined quartz tuning fork gyroscopes based on Coriolis acceleration. The vibration frequency of the sense tuning fork is equal to that of the drive tuning fork, and the vibration amplitude of the sense tuning fork is directly proportional to the vibration amplitude plus the angular velocity of the drive tuning fork. The output signals generated by the sense tuning fork through the piezoelectric effect are sensed by the external circuitry and amplified and demodulated to be a DC voltage signal proportional to the input angular rate. Further, the vibration phases of two tines of the fork in opposite directions can neutralize the acceleration effect. The tuning fork structure gyroscope is sensitive to the angular velocity only in one axis direction.

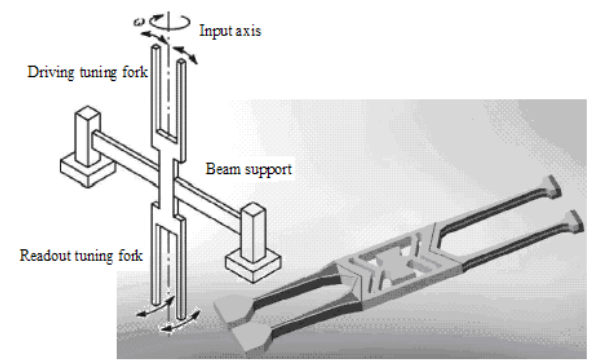

FIGURE I. STRUCTURE MODEL OF QRS BASED ON THE CORIOLIS ACCELERATION

\section{QRS DEVELOPMENT}

The quartz tuning fork micromachined gyroscopes emerge after a long period of researches and are developed on the basis of early tuning fork vibrating gyroscopes and crystal piezoelectric gyroscopes. The Sperry Gyroscope Company from USA proposed the principle of the tuning fork gyroscope in 1942, and reported their working prototype of the tuning fork angular rate sensor called Gyrotron with the sensitivity of $2^{\circ} / h$ only in 1953 , but was not put into mass production[3]. The metal vibrating beam gyroscope was produced by the General Electric through utilizing the piezoelectric quartz crystals. Later, Watson Industries designed a quartz gyroscope with the quartz crystal as the vibrating mass and the metal electrode excitation and detection. In 1980s, a number of other American and Japanese companies continued to research the tuning fork gyroscopes and develop the tuning fork gyroscopes for detection of bending vibration and the vibrating gyroscopes turning the torsional vibration into the bending vibration detection.

In 1991, Jan Soderqvist from the Uppsala University in Sweden reported their theoretical analysis, design and experimental results of QRS and the researches on the quartz micromachined gyroscopes began to increase rapidly. The 
quartz single-ended micro tuning fork was processed by the optical etching technique and the metal electrode was formed structurally by the special technologies to excite a vibration in the plane of tuning fork and to detect the angular velocity signals. Since 1986, the Systron Donner Inertial Division, a subsidiary of BEI Technologies, Inc., researched on the quartz rate senor and processed the $\mathrm{H}$-shaped double-sided tuning fork by mask etching. Every gyroscope has a specific integrated circuit chip to simultaneously compensate the errors caused by environmental changes, such as temperature and pressure. In 1990s the double-ended tuning fork gyroscopes spawned the QRS from researches in laboratories to the bulk production of practical and commercial applications [4]. At present USA, Japan, Sweden, France and other countries are doing researches on the quartz tuning fork micromachined gyroscopes [5]. Among them USA and Japan are leading countries with the most advanced technologies, mature processes, diverse structures and small size and their applications have been utilized in the automotive airbags, airplane aided navigation and tactical weapons.

In China, the research on the micromachined inertial devices started in 1994 and was covered by the National Micro Nano Pre-research Program in the "Ninth Five-Year Plan”. The research institutes of the QRS include No. 26 Institute of China Electronics Technology Group Corporation, Huazhong University of Science and Technology (HUST), National University of Defense Technology (NUDT), No. 704 Institute of China Aerospace Science and Technology Corporation and Beijing Institute of Technology (BIT) etc. [6-9]. HUST and NUDT have introduced innovations in the structural simulation and the process, studied the leveling process of crystal edge at sidewall after etching of quartz crystals, reduced the mechanical coupling error of gyroscopes and improved their precision. BIT has reported innovations in the design of drive and sense circuitry and signal processing. No. 26 Institute of China Electronics Technology Group Corporation has developed the prototype of quartz micromachined gyroscope in 2002 with the zero stability of $0.3^{\circ} / \mathrm{s}$, the most advanced in China at that time, and the engineering prototypes of the triaxis, single axis and quartz micrmachined gyroscopes in 2004 with the improved zero stability of no more than $0.2^{\circ} / \mathrm{s}$, but a relatively large size and weight.

\section{QRSS AND APPLICATIONS}

The quartz tuning fork micromachined gyroscopes developed by the BEI Systron Donner are one of the most advanced QRSs. For these gyroscopes, the minimum size is $8.12 \mathrm{~mm} \times 4.4 \mathrm{~mm} \times 0.25 \mathrm{~mm}$, and within the range of operating temperature, the standard range full scale is $\pm 1000^{\circ} / \mathrm{s}$, the nonlinearity is less than $0.05 \%$, the bandwidth is larger than $60 \mathrm{~Hz}$ and the zero stability is smaller than $0.01^{\circ} / \mathrm{s}$. The model QRS11 is a mature product in bulk production since 1990s and has been widely used in many systems, instruments and devices for stabilization, control, guidance and other functions. Fig. 2 shows the packaging and internal structure of QRS11. The performance parameters of this device are: the measuring ranges are $\pm 50^{\circ} / s, \pm 100^{\circ} / s, 200^{\circ} / s, \pm 500^{\circ} / s$ and $\pm 1000^{\circ} / s$, the bias stability of $100 s$ at constant temperature is $\leq 0.002^{\circ} / \mathrm{s}$, the resolution is $0.004^{\circ} / \mathrm{s}$, the weight is $60 \mathrm{~g}$ and the external dimension is $\phi=39 \mathrm{~mm}, H=17 \mathrm{~mm}$. This model has been used in Predator UAVs and air-to-ground Maverick missiles and the stabilization platform of the inertial system for Apache and Sikorsky S-92 helicopters. In 2003, the US Navy replaced the gyroscopes of the flight control systems of $A V-8 B$ and $F-18$ aircrafts to be QRS11. The quartz dual axis rate sensor QRS28 of BEI Technologies, Inc. was the advanced level and its external packaging and internal structure are shown in Fig.3. Table 1 gives the main performance parameters of this model.

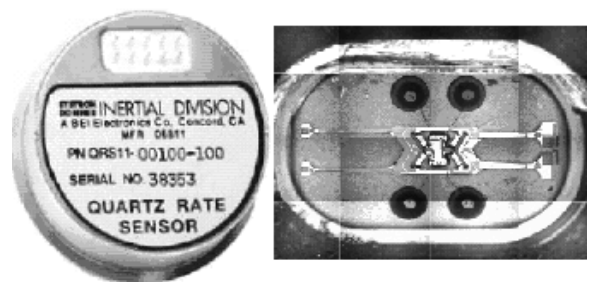

FIGURE II. QRS11 AND THE SENSITIVE ELEMENT STRUCTURE

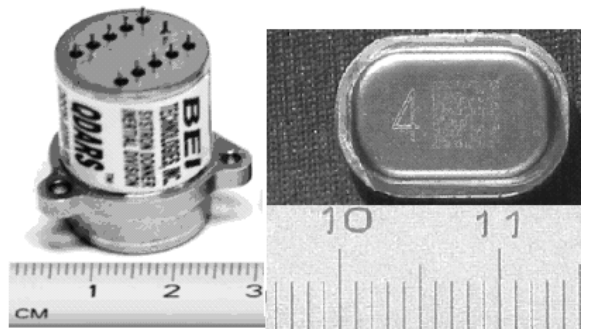

FIGURE III. QRS28 PACKAGE AND THE SIZE OF THE INTERNAL STRUCTURE

TABLE I. PERFORMANCE PARAMETERS OF QRS28 QUARTZ MICROMACHINED GYROSCOPE

\begin{tabular}{|l|l|}
\hline Power supply & $\pm 4.75 \sim 5.35 \mathrm{~V}(\mathrm{DC}) ;<50 \mathrm{~mA}$ \\
\hline Measuring range & $\pm 100 \%$ s; 200\% $; 400 \% / \mathrm{s}$ \\
\hline Output signals & $\pm 3.5 \mathrm{~V}$ \\
\hline Scale factor & $0.035 \mathrm{~V} / \%$; $0.0175 \mathrm{~V} / \%$; $0.00875 \mathrm{~V} / \% / \mathrm{s}$ \\
\hline Stability & $\pm 2 \%$ \\
\hline Zero-rate output & $<33 \mathrm{mV} 、 25^{\circ} \mathrm{C}$ \\
\hline Resolution & $<0.005^{\circ} / \mathrm{s}$ \\
\hline $\begin{array}{l}\text { Linearity (full-scale } \\
\text { range) }\end{array}$ & $<0.05 \%$ \\
\hline Bandwidth & $110 \pm 10 \mathrm{~Hz}$ \\
\hline $\begin{array}{l}\text { Range of operating } \\
\text { temperature }\end{array}$ & $-55 \sim 85^{\circ} \mathrm{C}$ \\
\hline $\begin{array}{l}\text { Maximum external } \\
\text { dimensions }\end{array}$ & $\begin{array}{l}\text { Diameter } 18 \mathrm{~mm}, \text { height } 26.7 \mathrm{~mm} \text { (sensitive } \\
\text { element: } 14.5 \times 10.0 \times 3.0 \mathrm{~mm}^{3} \text { ) }\end{array}$ \\
\hline Weight & $20 \mathrm{~g}$ \\
\hline
\end{tabular}

In Japan, Toyota Motor Corporation developed the doubleended tuning-fork quartz gyroscope in 2002 for applications in vehicle stability control, which adopted the beam instead of 
mass as the support parts. The tuning fork size is $15 \mathrm{~mm} \times 3.5 \mathrm{~mm} \times 0.3 \mathrm{~mm}$, as presented in Fig.4. For the gyro sensor, the standard range full scale is $\pm 100^{\circ} / \mathrm{s}$, the scale factor is $20 \mathrm{mV} /{ }^{\circ} / \mathrm{s}$, the linearity is smaller than $3 \%$ and the drift error is less than $1^{\circ} / s$ [10]. Seiko Epson Corporation and NGK Insulators Ltd. jointly developed the quartz vibratory gyroscope XV-3500CB measuring $5 \mathrm{~mm} \times 3.2 \mathrm{~mm} \times 1.3 \mathrm{~mm}$, mainly designed for use in digital cameras. In 2003, Nihon Dempa Kogyo Company developed a laminated quartz tuning fork measuring just $1.3 \mathrm{~mm} \times 2.2 \mathrm{~mm} \times 0.24 \mathrm{~mm}$. By bonding two monolithic wafers with reversing crystal directions, the quartz tuning forks have the same polarity of sidewall electrode in the sense mode and thus overcoming the manufacturing difficulty of split electrode at the tuning fork sidewall and greatly miniaturizing the gyroscope. With the hammer-headed structure, the range full scale is $\pm 240^{\circ} / \mathrm{s}$, the scale factor is $0.67 \mathrm{mV} /{ }^{\circ} / \mathrm{s}$, and the zero voltage is $1.349 \mathrm{~V}$. Owing to its small size, this product is mainly used in the consumer electronics field [11].

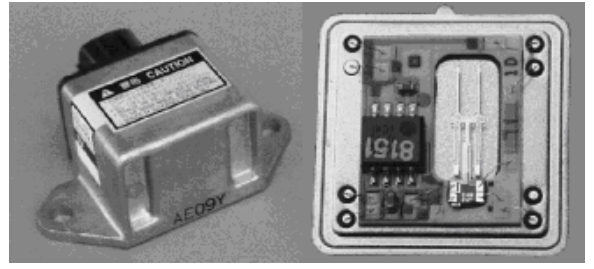

FIGURE IV

MIC-QUARTZ GYROSCOPE DEVELOPED BY TOYOTA MOTOR CORPORATION

No. 26 Institute of China Electronics Technology Group Corporation also successfully developed the double-ended tuning fork micromachined gyroscopes in 2003 by the wet etching process. It employs the in-plane vibration of the drive tuning fork as the drive mode and the out-plane vibration of the sense tuning fork as the sense mode. Its reported packaging dimension is $34 \mathrm{~mm} \times 24 \mathrm{~mm} \times 15 \mathrm{~mm}$, and the performance parameters of the single-axis gyroscope comprise: the range full scale of $\pm 100^{\circ} / \mathrm{s}$, the nonlinearity less than $0.04 \%$, the zero stability smaller than $0.2 \%$, and the shock resistance higher than $12000 \mathrm{~g}$. The National University of Defense Technology designed a framework hammer-headed micromachined quartz gyroscope in 2009, which was designed to be a framework double hammer-headed structure in 2010 making use of the high piezoelectric coefficient corresponding to the shear stress of quartz. This gyroscope uses the horizontal vibration of two tines as the drive mode and the vertical inplane vibration as the sense mode, without the need of split sense electrode made at the gyroscope sidewall. However, it employs not the conventional normal stress but the shear stress detection method to sense the changes in the $\mathrm{z}$-axis angular velocity and enhances the gyroscope sensitivity by optimizing the tamperness of the sense beam. For the gyroscope, the structural dimension is $13 \times 12.2 \times 0.5 \mathrm{~mm}^{3}$, the full-scale range is $\pm 150^{\circ} / \mathrm{s}$, the scale factor is $23.9 \mathrm{mV} /{ }^{\circ} / \mathrm{s}$, the nonlinearity is $1.1 \%$, the zero stability is $0.37^{\circ} / \mathrm{s}$, and the output noise floor is $0.1^{\circ} / \mathrm{s} / \sqrt{H z}$ 。

Many companies, universities and research centers of inertial devices from other countries conducted a lot of researches on MEMS IMU and made some achievements [8]. In Germany, aimed at the autonomous navigation for micro planes and based on the MEMS inertial sensors, S. Winkler et al. from Technical University of Braunschweig developed the small-size and lightweight MIMU and calibrated and tested it. The actual MIMU photo is presented in Fig.5.

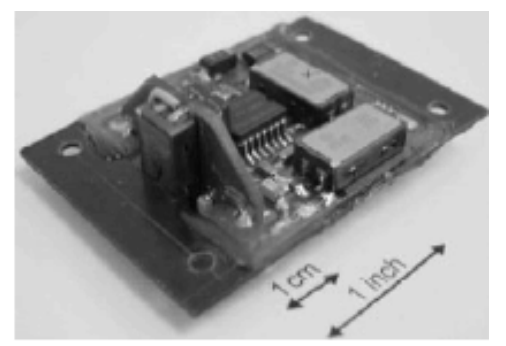

FIGURE V. MIMU DEVELOPED BY TECHNICAL UNIVERSITY OF BRAUNSCHWEIG

The Charles Stark Draper Laboratory has developed the MEMS IMU. For this device, the volume including the external case mounted part is $133 \mathrm{~cm}^{3}$, the weight is $272 \mathrm{~g}$, the input voltage is $5 D V C$, the overall $3^{\circ} / \mathrm{h}$ and $7 \times 10^{-5} \mathrm{~g}$, the drift is $0.05-0.09^{\circ} / \mathrm{h}$ over the temperature range of $0^{\circ} \mathrm{C} \sim$ $70^{\circ} \mathrm{C}$, the zero bias and the scale factor of one start-up is $8^{\circ} / \mathrm{h}$ and $2.5 \times 10^{-4}$ on average, and if the data in $1000 \mathrm{~s}$ is averaged, the zero bias stability reaches $0.1^{\circ} / \mathrm{h}$ for the IMU internal gyroscope.

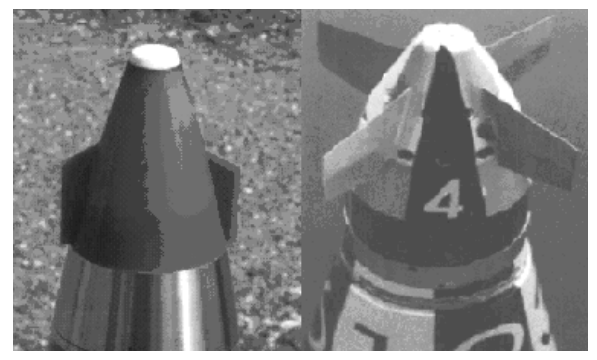

FIGURE VI.

TRAJECTORY CORRECTION FUZE AND AND CM-ATD BOMB

The MIMU of the Draper Lab has already completed a series of experiments in miniature weapon systems, inclusive of: (1) successful experiments in extended range guided munitions, and the size of the INS/GPS, computer and circuit is $76.2 \mathrm{~cm}^{2}$; (2) applications in advanced EX171 weapons for the US Navy and XM982 weapons for the Army, and the IMU with the diameter of $50.8 \mathrm{~mm}$ and the height of $30.5 \mathrm{~mm}$ can withstand the gunfire acceleration of $16000 \mathrm{~g}$ with the power consumption of $2 W$ and the cost of 1500 US dollars; (3) experiments in space shuttles and X-33, to be used in micro satellites. Ever since 1990s, the strapdown inertial navigation 
systems have been widely used for missile guidance as shown in Fig.6. Its correction system comprises the General Data Transfer Platform (GTP), the Inertial Measurement Unit (IMU), the Canard Actuation System (CAS) and the navigational computer and energy [12].

\section{TEChNOLOGY ReVIEW OF QRS}

The quartz tuning fork micromachined gyroscopes is a kind of vibratory gyroscopes and does not need complication structure and circuitry for control and detection owing to good piezoelectric effect of quartz. The mature wet etching process is utilized, but the sidewall needs fabricating split electrodes and there is the problem in coupling of drive vibration and sense vibration. The key challenges to be overcome are as follows. (1) The quartz crystals are processed by the wet etching process, the processed devices encounter the crystal edge at the sidewall and has the uneven sidewall, and hence the mechanical coupling error and the orthogonal error occur to gyroscopes. (2) In order to achieve the optimum performance of the quartz micromachined gyroscope, the drive mode and the sense mode must be matched. This imposes high requirement for structural symmetry of the quartz tuning fork and requires precise processing means and methods. (3) The quartz tuning fork micromachined gyroscope needs to fabricate split electrode at sidewall. This restricts the miniaturization of its size. (4) The material characteristics of quartz crystals result in the piezoelectric effect completely unavailable in z-axis direction. It is only the single-axis measurement. The application in the multi-axis sense can be achieved through the orthogonal combination.

The confined etchant layer technique (CELT) is utilized to flatten the edge at $\mathrm{X}$-axis towards the center due to chemical corrosion of quartz. The single-point fixed packaging of the double tuning forks is good for the stability of operation performance of quartz tuning fork. The selection of packaging case, the packaging method and the fixing process of quartz tuning fork directly affect the performance of micromachined quartz gyroscopes. Temperature compensation and vacuum package are the main measures taken for improving the signalto-noise ratio of QRSs. The laser technique is utilized for fabricating and cutting the electrode layer of quartz tuning fork, balancing the mass to adjust the tine symmetry, improving impedance performance of tuning forks, increasing $\mathrm{Q}$ of quartz tuning forks and enhance the yield rate and accuracy of micromachined quartz tuning forks.

\section{CONCLUSIONS}

Ever since 1990s, the quartz tuning fork micromachined gyroscopes demonstrate very promising commercial applications for industrial and traffic automation, mainly for navigation, vehicle attitude control and absolute angular velocity. They have been broadly applied in the fields such as automotive airbag, automotive yaw and rollover detection, GPS-aided navigation and guided munitions. Moreover, the future weapon systems will obviously develop from the large strategic systems to the miniaturized tactical systems. The micro inertial systems and the micro inertial instrument technologies are the direct outcomes of this trend. The quartz tuning fork micromachined gyroscopes are developing towards the integrated inertial measurement units (IMUS), namely the strapdown inertial navigation systems integrated the accelerometer and gyroscope with the measurement circuits[13]. They can be used separately or together with the global positioning system / inertial navigation system (GPS/INS)[14]. After further improvement in size and accuracy, they will become increasingly important on the customer electronics, biological, medical, industrial machinery and military markets.

\section{REFERENCES}

[1] S. F. Konovalov, A. V. Kuleshov, V. P. Podtchezertsev, et al. Vibrating angular rate sensor [J].Integrated Navigation Systems Proceedings of 10th International Conference, St Petersburg,2003,107-117.

[2] HE Haiyang, WANG Zhangbo. Design of Low-cost Inertial Navigation System Based on MEMS Gyroscope [J]. Metrology \& Measurement Technology, 2014,4:34-37.

[3] Baker G. N. Quartz rate sensor from invention to application. In Proceedings of DGON Symposium Gyro Technology. Stuttgart. 1992, 161-166.

[4] Silva N.R., Murray W.G. Low cost quartz rate sensors applied tactical guidance IMUs. IEEE Proceedings of Position Location \& Navigation Symposium, 1994. 37-42.

[5] MEGHERBI S, LEVY R,PARRAIN F, et a1. Behavioral modeling of vibrating piezoelectric microgyro sensor and detection electronics[C]. Proceedings of International Conference on Thermal, Mechanical and Multi-Physics Simulation Experiments in Microelectronics and MicroSystems. London, UK, 2007:1-4.

[6] ZHANG Qiaoyun, Lin Ri-le, XIE Jia-wei, et al. Application of Quartz Micromachining to Realization of Micro-gyro [J]. Piezoelectrics \& Acoustooptics, 2009,31(6): 788-790.

[7] ZHAO Ke,MA Yuefei,CUI Fang, et al. Experimental Research on Micro-Electroplating Process to Adjust Mass Balance of Micro-Quartz Gyroscope[J]. Transactions of Beijing Institute of Technology, 2013, 33 (9):985-990.

[8] LIN Rile, LI Wenyun, ZHAO Jianhu, et al. Optimization Research on Anti High Shock Ability of Quartz MEMS Gyroscope[J]. Piezoelectrics \& Acoustooptics, 2013,35(1):56-68.

[9] XIE LiQiang,XING Jianchun,DONG Pei, et al. Research on the Exciting Control Method for Micro- machined Quartz Gyroscope[J]. Acta Armamentarii, 2013,34(6):770-775.

[10] NONOMURA Y, FUJIYOSHI M, OMURA Y. et a1. Quartz rate gyro sensor for automotive control [J]. Sensors and Actuators: A, 2004,110 (1/2/3):136-141.

[11] SATO K, ONO A, TOMIKAWA Y. Finite element method simulation of double-ended tuning fork quartz resonator for application to vibratory gyro-sensor[J]. Japanese Journal of Applied Physics, 2003,42: 3115 3119.

[12] MASAKO T. An industrial and applied review of new MEMS devices features [J]. Microelectronic Engineering, 2007, 84: 1341-1344.

[13] Madni A.M., Costlow L. E., Knowles S.J. Common design techniques for BEI GyroChip quartz rate sensors for both automotive and aerospace/defense markets[J].Sensors Journal, IEEE. Oct. 2003, 3(5):569-578.

[14] HE Haiyang, WANG Zhangbo. Design of Low-cost Inertial Navigation System Based on MEMS Gyroscope [J]. Metrology \& Measurement Technology, 2014,4:34-37. 